This is the postprint version, according to green open access requirements, of the following article published in "Disability and Rehabilitation: Assistive Technology", as:

\title{
Clinical validation of a novel postural support device for hospitalized sub-acute post-stroke wheelchair users.
}

\author{
Jan F. Veneman, Je Hyung Jung, Iraitz Manterola del Puerto, Maria Luz Seco \\ Rodriguez, Iñaki Camiruaga Zalbidea, Sergio Cardoso Martin, Lorea Mendoza \\ Arteche \& Jorge Alemany Herrera
}

To cite this article:

Jan F. Veneman, Je Hyung Jung, Iraitz Manterola del Puerto, Maria Luz Seco

Rodriguez, Iñaki Camiruaga Zalbidea, Sergio Cardoso Martin, Lorea Mendoza Arteche \& Jorge Alemany Herrera (2016): Clinical validation of a novel postural support device for hospitalized sub-acute post-stroke wheelchair users, Disability and Rehabilitation: Assistive Technology, DOI: 10.3109/17483107.2016.1159739

To link to this article: $\underline{\mathrm{http}: / / d x . d o i . o r g / 10.3109 / 17483107.2016 .1159739}$

\begin{abstract}
Purpose:

We present a novel wheelchair posture support device (WPSD) and its clinical validation. It was developed in order to assure correct sitting posture and to reduce caregiver time spent for repositioning of wheelchair-bound hospitalized post-acute stroke patients.
\end{abstract}

Method:

The device was validated with 16 subjects during a period of five days in which use of the device was compared with regular care practice.

Results:

The device was used for the five consecutive days in $69 \%$ of patients, while for $6 \%$ it was not suitable; $25 \%$ did not complete the five days for reasons unrelated to the device. Caregivers needed to re-position the patients that used the device for the full five days $(n=11)$ on average $52 \%$ less times when using the device, as compared to regular practice. Furthermore, the device was rated as usable and functional by the caregivers while significantly reducing perception of trunk pain in patients during the use of it.

Conclusions:

The newly designed WPSD is a valuable system for the improvement in medical assistance of hospitalized wheelchair-bound post-stroke patients by reducing pain and number of re-positioning manoeuvres. The WPSD might be applicable to any group of patients who need posture control in either wheelchair or common chair with arms support. 


\title{
Clinical validation of a novel postural support device for hospitalized sub-acute post-stroke wheelchair users.
}

\author{
Jan F. Veneman, Je Hyung Jung, Iraitz Manterola del Puerto, Maria Luz Seco \\ Rodriguez, Iñaki Camiruaga Zalbidea, Sergio Cardoso Martin, Lorea Mendoza \\ Arteche \& Jorge Alemany Herrera
}

\section{Introduction}

Cerebral Vascular Accident (CVA) or stroke is the second leading cause of disability worldwide, after dementia. As a reference, in 2010, an estimated 16.9 million incidences of stroke occurred, 33 million prevalent stroke cases and 5.9 million stroke deaths were reported, and 102.2 million disability-adjusted life years were lost [1]. A substantial percentage of acute stroke patients is hospitalized and subsequently discharged to short-term rehabilitation hospitals or to long-term care institutions. The burden of stroke will only rise over coming decades because of demographic transitions of populations, particularly in developing countries. Survivors normally show a degree of functional recovery after their stroke, and this recovery is typically supported by rehabilitation care, depending on the severity of the stroke and the local health system. Taken together, these facts pose a heavy and growing burden on health service providers worldwide. For this reason, ways are sought to increase efficiency and effectivity of the provided care.

During the acute and sub-acute phases after stroke, proper positioning of the patient is considered of paramount importance. Upright positioning like seating or standing is shown to be beneficial on a number of aspects. During the acute phase, important effects are the support of cerebral oxygenation, maintaining nutrition and hydration, as well as psychological benefits [2] [3] [4]. For the sub-acute phase, the main benefits of upright positioning are related to supporting muscle tone and promoting motor system recovery [5]; control of trunk posture while sitting is considered to be the first step in motor rehabilitation [6]. For practical reasons, during the sub-acute institutional phase, wheelchairs are widely used for upright sitting as well as mobility.

Stroke patients, however, typically suffer from hemiparesis and other motor control difficulties. Often, especially in acute and sub-acute phases, they are not able to maintain a proper sitting posture on a wheelchair [6]. Because these patients spend a considerable amount of time per day in a wheelchair, it is important that they are able to correctly sit on the wheelchair without adverse effects that may originate from a poor sitting posture. Typical adverse effects of improper sitting are sensation of discomfort, development of muscular pain, development of back or shoulder injuries and related pain, and pressure ulcers [7]. Additionally, in severe patients there is a risk of falling out of the wheelchair. The clinically recommended proper sitting posture is characterized by: laterally symmetric posture, upright trunk posture and hip and knee angles of around 90 degrees [8].

In a rehabilitation hospital, where patients temporarily live during the sub-acute stage, the following methods and products are typically being used to assure safe and correct seating during wheelchair use:

- Manual re-positioning by nurses, based on observation of posture

- Restraints, such as belts, mainly to prevent falling out of wheelchair

- Specifically designed cushions and pillows, for example a 'pommel cushion', to correct sitting posture

- Table mounted on wheelchair that supports the arms

In some cases, specific high-end backward-tilting wheelchairs are being used [2] [7]. 
In the Hospital Gorliz ${ }^{1}$, which was participating in this project, the common method is to have nurses re-positioning patients when needed. Additionally, a (transparent) table is used in the wheelchair in order to better position the hands, arms and shoulders. Belt-type restraints are used when needed for safety. Main problem with this way of working is that it does not effectively prevent an improper sitting posture so that it is required to (re-)position the patient, which is very time consuming and laborious, especially with severe stroke patients.

In order to improve this practice, we developed a new type of positioning device for use in a basic wheelchair, intended for sub-acute stroke patients. The main goal of this device is to reduce the needed number of re-positionings (and thus nursing time) per patient, while achieving and maintaining a safe, comfortable and rehabilitation-supporting sitting posture. The device should not completely block the movement of the patient, but leave some space for trunk movement, both for comfort (e.g. respiration) and to challenge the patient and thus train proper positioning by encouraging their own remaining trunk control.

In this paper we explain the user-centred design of this device to achieve the goals and realization of the device, which is based on a novel variable stiffness material, called Varstiff@ [9] [10]. The validation is based on the use of prototypes of the device in Hospital Gorliz, while measuring the amount of caregiver time, usability and user comfort perception. First, we describe the development of the Wheelchair Posture Support Device, followed by the methods and results of the validation ${ }^{2}$. Finally, the discussion and conclusion are presented.

\section{Development of the Wheelchair Posture Support Device based on Varstiff@ through a user-centred design process}

The idea for a Wheelchair Posture Support Device (WPSD) to assists sub-acute post stroke patients in maintaining correct upright posture in a wheelchair originated from several interviews with medical doctors and physiotherapists in Hospital Gorliz on identifying potential applications of the Varstiff(C) technology.

\section{Requirements}

The collected requirements for the device after the interviews with Hospital Gorliz clinical staff are divided in functionality and usability requirements, listed below.

Functionality requirements of the WPSD

- It should facilitate the $90^{\circ}$ rule [8], which indicates that the patient should sit with hips, knees and ankles flexed at $90^{\circ}$, in other words, prevent large inclination of a patient in side directions as well as forward direction while it allows small movement in all directions to avoid pressure ulcers.

- $\quad$ It should prevent a patient from sliding down out of the wheelchair.

- $\quad$ It should prevent pain in trunk and shoulder during the use.

- $\quad$ It should allow proper respiration during the use.

Usability requirements of the WPSD

Cleanability: It should easily be cleanable with antiseptic as it is shared between different patients in hospital.

\footnotetext{
${ }^{1}$ Hospital Gorliz, Urezarantza Ibiltokia, 1, 48630, Gorliz, Vizcaya, Spain

2 The basic ideas of the Wheelchair Posture Support Device were earlier presented at the TAR 2015 conference: Technically Assisted Rehabilitation [16], where the evaluation of a WPSD with one patient was reported.
} 
- Adaptability: It should encompass different body sizes of users, normally with fat accumulation in the trunk, which is a common body characteristic of the target group.

- $\quad$ Safety: All edges should be rounded and not sharp.

- Manipulability: It should facilitate simple and easy manipulation by either clinical staff or family caregiver whereas it should not allow users to change status of the device.

\section{Overall system development through a user centred design process}

Starting from these requirements, iteratively a number of prototypes was made and evaluated with clinical users from Hospital Gorliz, in order to realize a user-centred design process and an eventual prototype that would be usable for a clinical validation study. The resultant designed system consists of five main elements: trunk support, seat cushion, connection element to wheelchair, table, and portable electrical pump. The trunk support prevents the trunk from excessive bending forward as well as to the right and left sides while allowing proper respiration and movement. This main function is assured by the application of Varstiff@ technology, which allows shaping of a supportive structure to individual needs in a flexible state, and then can be switched to a rigid state in a decided shape. The description starts with background information on Varstiff@ as the material that facilitates to fulfil the design goals. After that the design is described in more detail.

\section{a. Description of Varstiff ${ }^{\odot}$}

'Varstiff ${ }^{\mathrm{O}}$ ' is a 'material' that is able to switch from a flexible textile-like state to a more rigid state (similar to HDPE) by applying negative pressure (vacuum). This 'material' consists of a laminate of flexible textile layers integrated in a hermetic flexible envelope suitable for pressure regulation (Figure 1). When applying a vacuum, the layers are compressed, which decreases their mutual distance and increases friction between them. This in turn increases the stiffness of the $\operatorname{Varstiff}^{\odot}$ as a material. Flexibility as well as rigidity under vacuum varies with material characteristics of layers and the number of layers. Details of Varstiff $^{\odot}$ are described in [11]. Information can also be found in the patents that were filed [9] [10].

(a)

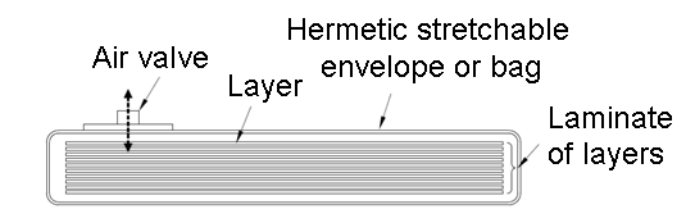

(b)

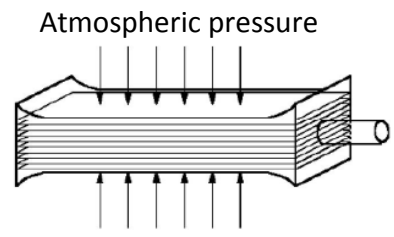

(c)

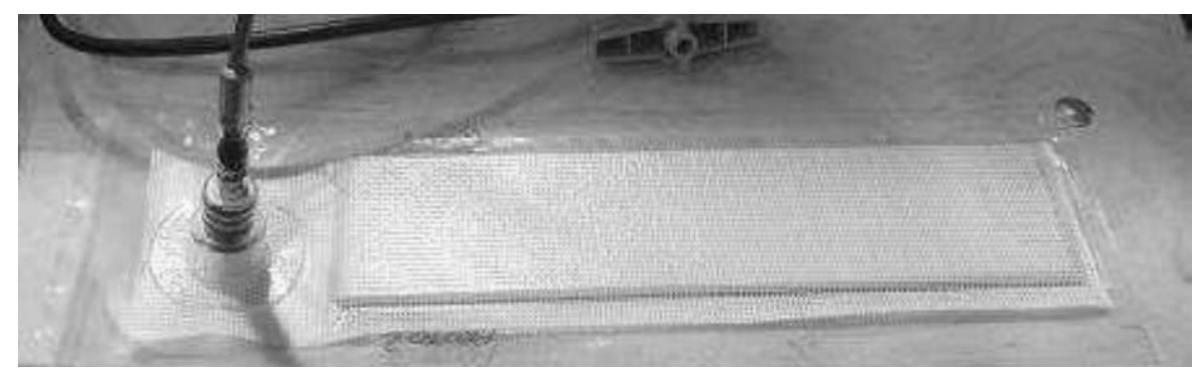

Figure 1: Varstiff ${ }^{\odot}$ 'material'; several representations of a sheet of Varstiff ${ }^{\odot}$, consisting of a hermetic bag, containing a number of layers. (a) Schematic representation of a Varstiff ${ }^{\mathcal{O}}$ sheet, (b) $3 D$ representation of a Varstiff $f^{\circ}$ piece, with layer compression due to vacuum inside the bag, (c) rectangular sample of Varstiff ${ }^{\odot}$ with air evacuation valve under vacuum. Used with permission from [10]. 


\section{b. Detailed design and implementation}

Thanks to Varstiff ${ }^{\odot}$ properties, the trunk support enables to switch its state from flexible to rigid and vice versa. In the flexible state, the trunk support is easily conformed to patient's trunk; once the support is placed in the correct position, it is rigidified by a vacuum pump. The conceptual design of the trunk support is shown in figure 2 . The Varstiff ${ }^{\odot}$ contains nine inner layers made of the patented composition [9]. The dimension of the trunk support was decided in accordance with human anthropometric data [12], as well as feedback from clinicians in Hospital Gorliz. A manual operated valve was welded in order to evacuate the air. A vest made of soft and skin-friendly material was added to prevent direct contact of skin to the outer bag.
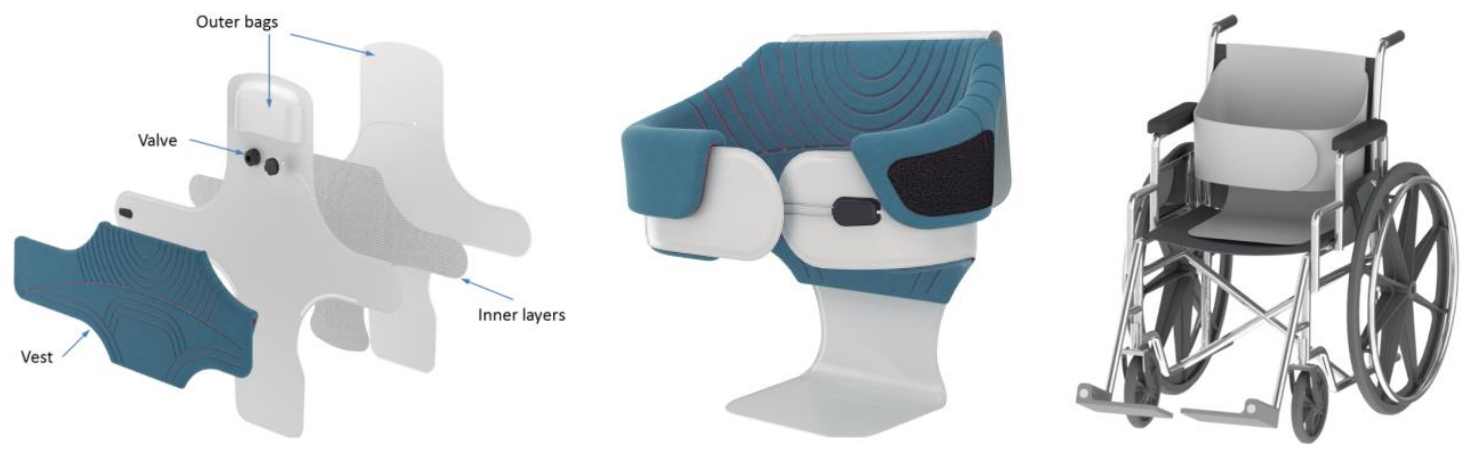

Figure 2: Trunk support of the Wheelchair Posture Support Device. 3D design (Left figure) shows the trunk support, containing the Varstiff ${ }^{\odot}$ material, and it's covering washable comfortable vest. The rightmost picture shows the trunk support alone inside a wheelchair.

The actual trunk support, including additional parts, as was implemented is shown in Figure 2 and 3. The vest shown in the conceptual design was replaced with flexible forms which are directly attached to the main cushion for simplicity of implementation. As closing mechanism of right and left segments, a plastic buckle was utilized.

The seat cushion is composed of two parts, one is a silicone seating cushion and the other a soft pad that is placed between thighs (also called 'pommel cushion') (Figure 3). The silicone cushion provides comfortable sitting while the soft pad prevents excessive hip abduction, which is likely to result in incorrect sitting posture.

The trunk support is connected to the wheelchair through an element that is placed between handlebars of the wheelchair. It aims to maintain the trunk support in initial position which is set by the caregiver. In addition, it allows the patient's limited movement in front, right and left directions instead of completely restraining the patient.

A connective element was designed to secure the trunk support to the wheelchair. This is a type of belt made of a non-elastic textile. It has a buckle for easy connection/disconnection to the wheelchair handles and a hook that is used to secure the trunk support. High friction materials are used for contact surfaces to further prevent sliding of the trunk support inside the wheelchair.

The supportive table plate is a part that was already in use in Hospital Gorliz, in order to support the hemiplegic upper limb. As shown in figure 3 , it is a square table made of transparent plastic material and is placed on the armrests of the wheelchair. The affected arm is positioned on the table and the patient is asked to try to keep the (possibly) spastic hand open on the table. The table aims to maintain the affected arm in correct position, supporting upright position and correct shoulder position. It's worth noting that only use of the table is not enough to achieve the aims as affected hand and arm easily change their originally set location due to unintended motion of the patient. 
A prototype portable electrical pump unit is included to evacuate air from the trunk support in order to make the trunk support rigid enough to maintain upright posture. The pump unit can be handheld and consists of a vacuum pump, valves, a battery, and a battery level indicator. It enables to reach a negative pressure of 700 mbar which is required for the trunk support to have an adequate rigidity, preventing large deformation of the WPSD caused by the patient's trunk motion.
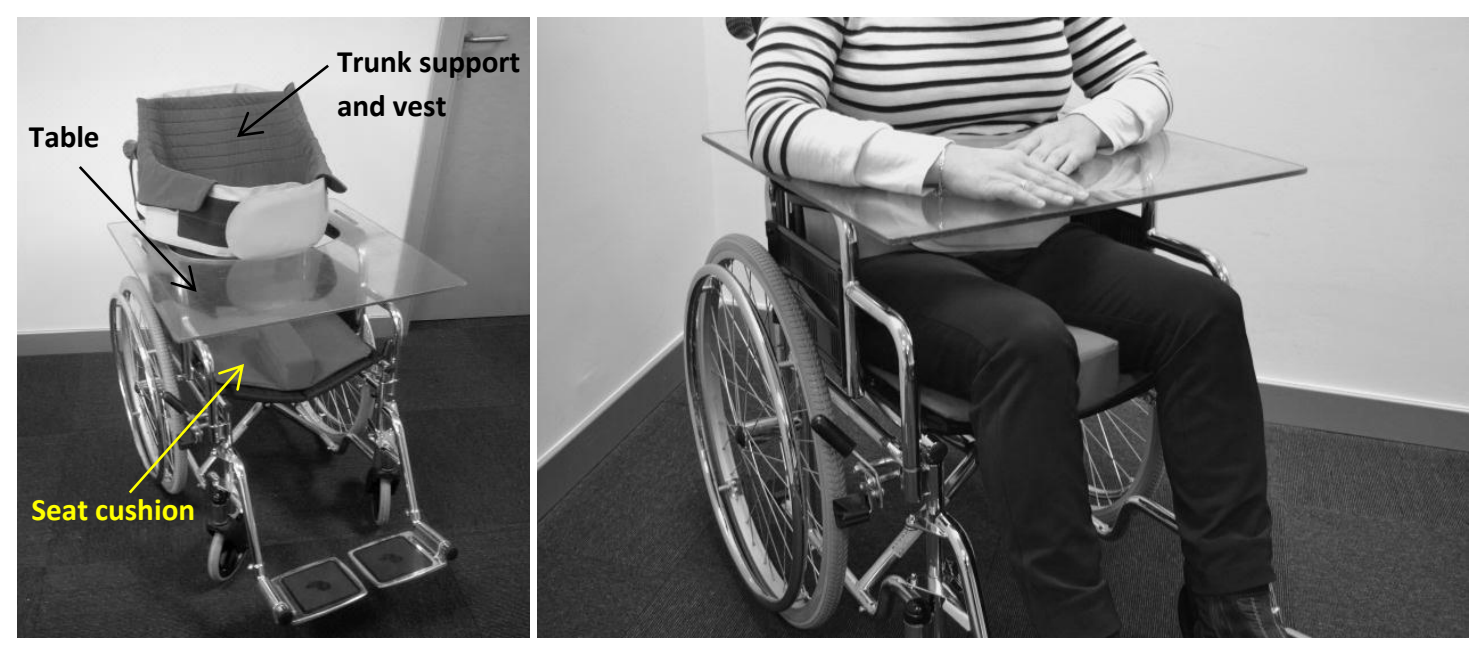

Figure 3: Final prototype of the Wheelchair Posture Support Device. Left: WPSD installed in a wheelchair - trunk support containing Varstiff ${ }^{\odot}$ covered with a 'vest', seat cushion with 'pommel', transparent arm support table. Right: Person seated with a correct posture due to WPSD and transparent table.

\section{Procedure of operation}

The procedure of operating the WPSD is:

- Connect the WPSD to the wheelchair with straps

- Help the patient sit down in the wheelchair equipped with WPSDShape the device (in flexible state) to the size and shape of the patient

- Attach the supportive table when needed

- $\quad$ With the vacuum pump, provide a vacuum through the back valve

- Provide the normal activities to or by the patient

- Re-position the patient in case of a poor sitting posture

- Re-apply the vacuum and shaping when needed, by opening and closing the valve

\section{Validation of the Wheelchair Posture Support Device based on Varstiff ${ }^{\complement}$}

After fabricating a number of prototypes based on the described design, a validation study was performed in Hospital Gorliz. First we describe the methods of this study, followed by the results.

\section{Methods:}

For comparison of the situation between using the device and using the conventional methods, a single-subject design was selected, where subjects serve as their own control. The advantage of such a design is that it allows demonstrating effects that are not visible when group based statistics are used, especially when there are large variations between subjects - as can be expected in this study.

The evaluation period for each subject was five consecutive days, from Monday to Friday. This period was considered adequate because the status of this type of patients may evolve rapidly and 
their situation while sitting might change considerably from one week to the next. Each patient tested the prototype three hours per day during five days, alternating periods using the prototype and routine caregiving practice without using the device on the same day, and daily alternating mornings and afternoons of use during on subsequence days to avoid influences.

Table 1 shows a typical Wheelchair Posture Support Device usage sequence for a patient.

\begin{tabular}{|l|l|c|c|c|c|c|}
\hline & & Day 1 & Day 2 & Day 3 & Day 4 & Day 5 \\
\hline $\begin{array}{l}\text { Sequence } \\
\text { option } 1\end{array}$ & Morning (3 hrs) & WPSD & Usual care & WPSD & Usual care & WPSD \\
\cline { 2 - 7 } & Afternoon(3 hrs) & Usual care & WPSD & Usual care & WPSD & Usual care \\
\hline $\begin{array}{l}\text { Sequence } \\
\text { option } 2\end{array}$ & Morning (3 hrs) & Usual care & WPSD & Usual care & WPSD & Usual care \\
\cline { 2 - 7 } & Afternoon(3 hrs) & WPSD & Usual care & WPSD & Usual care & WPSD \\
\hline
\end{tabular}

Table 1: The randomly assigned two optional sequences of Wheelchair Posture Support Device ('WPSD') use and usual care during the WPSD validation in one patient during five consecutive days. Both options were randomly assigned as there may be structural difference between morning and afternoon behaviour, for example due to fatigue

A total of sixteen patients were included in the study. For subject recruitment the following inclusion criteria were established:

- Patients admitted in Hospital Gorliz

- Patients with difficulties to maintain upright position of the trunk while sitting in a wheelchair

- Informed consent signed by the patient or his/her legal representative

Patients that could not be seated on a chair were excluded for this study. The study started in October 2013 and finished in August 2014.

\section{Data Collection:}

Per included subject the following demographic and clinical data was collected: gender, age, constitution (underweight, normal, obese, according to Body Mass Index (BMI)), weight, height, pathology, admission date, date when injury occurred.

During the study as primary variables were collected: a) the feasibility to use the device for five half days in one week according to the study's usage protocol, b) the number of re-positioning incidences; time, date and name of the healthcare professional performing the re-positioning, and c) perceived pain; pain in this type of patients is usually a postural pain that can be classified in shoulder postural pain and trunk postural pain [13] [14]. Patients with sufficient cognitive skills answered using a 10 grade visual scale ranging from 'no pain' to 'unbearable pain' [15]. In the rest of the cases, healthcare professionals assessed this variable interpreting patient's gestures, as it is done in common clinical praxis, d) perceived comfort; patients with sufficient cognitive skills answered this question by means of a 10 grade visual scale. In the rest of the cases, healthcare professionals assessed this variable interpreting patient's gestures, as it is done in common clinical praxis.

Each of these variables was collected every day / every session of use of the device, and then averaged per patient over the five days of use in order to obtain the score for that specific patient. 
As secondary outcome, the opinions of the professional caregivers operationally involved in the study were collected. This was split in two aspects:

a) Ease of use of the prototype was scored on a numeric scale from 1 to 5 (from easiest to most difficult) on the following aspects 'ease to put the prototype on the wheelchair', 'ease to apply the device to the patient', 'ease to apply vacuum to the device', 'ease to re-position the patient', 'ease to take out the prototype from the wheelchair', 'hygiene', b) functionality; functionality of the prototype was scored on several aspects, scored on predefined options per aspect and scored per application of the WPSD on a specific patient. The scored aspects of functionality were: 'rigidity for support', scored as 'adequate' versus 'need of repeated application of vacuum' versus 'insufficient'; 'adaptability of shape to patient', scored as 'adequate' versus 'insufficient at some places' versus 'overall insufficient'; 'difficulties while transferring patients from bed to wheelchair and vice versa', scored as 'with difficulties' versus 'without difficulties' 'time needed to perform a re-positioning', scored as 'low' versus 'medium' versus 'high'.

The primary data collected within the study were anonymized and identified by a code (G+number, e.g. G01), so that the identity of the patient was not disclosed to any person, unless when one of following situations occurred: medical emergency situation, requirement of healthcare administration or legal requirement. Treatment, communication and transfer of personal data of patients participating in the study were done according to the Organic Law 15/1999 of 13 December on the Protection of Personal Data.

The data relevant to this study were collected by the caregivers of Hospital Gorliz by means of a Case Report Form (CRF) located at the back of the wheelchair within an opaque bag.

\section{Data analysis:}

Qualitative or categorical variables, such as sex, constitution or characteristics of the device, were described as percentage of total, whereas quantitative variables (mainly number of re-positioning manoeuvres, comfort, trunk pain and shoulder pain) were analysed by computing the mean and standard deviation. The Shapiro-Wilk test was used to test the normality of each set of quantitative data, for both "with WPSD" and "without WPSD". Differences between groups (with and without WPSD) were contrasted by means of the $t$ of Student test. Statistical differences were further tested by using the nonparametric Mann-Whitney $U$ test. A confidence interval of $95 \%$ was used, with a significance level of $5 \%$ ( $p$-value of 0.05). For data analysis, the SPSS 19.0 software was used.

\section{Ethical considerations:}

The protocol of the study was written in May 2013 and was submitted for ethical approval to the Ethical Committee of the Cruces University Hospital, which issued a favourable opinion in July 2013. In June 2013, the protocol was also sent to the Spanish Agency of Medicines and Medical Devices (AEMPS), which determined that the device under study was not classified as a medical device.

No patient was included in the study and no procedure related to the study was performed before patients and family caregivers were given oral and written explanations about the study and the informed consent was signed.

\section{Results}

\section{Demographic characteristics of participants}

Sixteen patients were recruited for participation in the study, of which nine male $(56 \%)$ and seven female (44\%), with ages ranging from 40 to 86 years (69.3 SD 12.1). Most of the participants (44\%) had a normal constitution, followed by underweight participants (37\%) and overweight (obese) participants (19\%). The lightest constitution was $50 \mathrm{~kg}$ at $156 \mathrm{~cm}(\mathrm{BMI}=20.55)$; the heaviest constitution was $79 \mathrm{~kg}$ at $150 \mathrm{~cm}$ (BMI=35.11). Except for the youngest participant (40 years old) that suffered a generalized hypoxia, all participants had suffered a stroke episode. 


\section{Applicability of WPSD for included participants.}

Of the sixteen patients, admitted to the study, eleven finished the study as planned. Five patients used the device for a shorter period than the planned five days, for various reasons:

- One patient quit after two days because the device was not adequate for this patient; even with the device patient could not maintain a proper seating position, mainly caused by sliding of the WPSD inside the wheelchair

- One patient quit after three days because of transfer to another hospital for urgency treatment unrelated to the study

- One patient quit after four days, translated to another hospital for urgency treatment unrelated to the study

- One patient quit after four days because of pain and discomfort and was diagnosed with a back lesion unrelated to the study

- One patient quit after four days because family bought a specially adapted chair, which did not allow use of the device under study.

This means that of the sixteen patients fulfilling the inclusion criteria, for 1 patient (6\%) the device was not suitable, 4 patients $(25 \%)$ used it shorter than planned due to reasons unrelated to the device, and 11 patients (69\%) completed the follow-up of 5 days successfully.

The statistics that compare the situation without posture support and the situation with posture support are based on the $69 \%$ of included participants that indeed completed the study fully, as intended.

\section{Results of Analysis}

\section{Primary outcomes}

In the patients that completely fulfilled the follow-up of five days $(n=11)$, usage of the device resulted in an average reduction in number of re-positionings per half day from 4.6 to 2.2 (reduction of $52 \%$ ), an increase of perceived comfort from 3.9 to 6.9 (increase of $77 \%$ ), a decrease of perceived shoulder pain from 3.4 to 2.5 (decrease of $26 \%$, no statistically significant) and a decrease of perceived trunk pain from 4.7 to 3.7 (decrease of $43 \%$ ). All these changes were statistically significant, considering the group of eleven that finished the study, except for the shoulder pain result. Detailed results are shown in table 2 and in figure 4.

\begin{tabular}{|c|c|c|c|c|c|c|c|c|c|c|c|}
\hline \multirow{2}{*}{\multicolumn{2}{|c|}{ Parameter }} & \multirow{3}{*}{\begin{tabular}{|c|}
$\begin{array}{c}\text { Experimental } \\
\text { parameter value }\end{array}$ \\
Mean \pm SD \\
2.2227 SD 1.2485 \\
\end{tabular}} & \multicolumn{2}{|c|}{ Shapiro-Wilk test } & \multicolumn{4}{|c|}{ t-test } & \multicolumn{2}{|c|}{ Mann-Whitney test } & \multirow{2}{*}{$\begin{array}{c}\text { Interpretation } \\
p=0.05(5 \%)\end{array}$} \\
\hline & & & $\mathbf{w}$ & $p$ & Mean & 95\% confid. & $t$ & $p$ & $\mathbf{U}$ & $p$ & \\
\hline \multirow{2}{*}{$\begin{array}{l}\text { Number of } \\
\text { repositionings } \\
\text { per half day }\end{array}$} & WD & & 0.9077 & 0.2293 & \multirow{2}{*}{2.4167} & \multirow{2}{*}{$\begin{array}{c}0.7104- \\
4.1229\end{array}$} & \multirow{2}{*}{-2.9545} & \multirow{2}{*}{0.0078} & \multirow{2}{*}{18} & \multirow{2}{*}{0.0056} & \multirow{2}{*}{$\begin{array}{c}\text { Statistically } \\
\text { significant } \\
\text { difference }\end{array}$} \\
\hline & WOD & 4.6394 SD 2.4085 & 0.9344 & 0.4567 & & & & & & & \\
\hline \multirow{2}{*}{$\begin{array}{l}\text { Comfort } \\
\text { Rating }\end{array}$} & WD & 7.8544 SD 2.0141 & 0.8531 & 0.2135 & \multirow{2}{*}{2.9818} & \multirow{2}{*}{$\begin{array}{c}1.3205- \\
4.6431\end{array}$} & \multirow{2}{*}{-3.7441} & \multirow{2}{*}{0.0013} & \multirow{2}{*}{16.5} & \multirow{2}{*}{0.0042} & \multirow{2}{*}{$\begin{array}{c}\text { Statistically } \\
\text { significant } \\
\text { difference }\end{array}$} \\
\hline & WOD & 4.8727 SD 1.7089 & 0.9172 & 0.2962 & & & & & & & \\
\hline \multirow{2}{*}{$\begin{array}{l}\text { Trunk Pain } \\
\text { Rating }\end{array}$} & WD & 2.6727 SD 1.7211 & 0.8827 & 0.1125 & \multirow{2}{*}{2.0027} & \multirow{2}{*}{$\begin{array}{c}0.4278- \\
3.5776\end{array}$} & \multirow{2}{*}{-2.6526} & \multirow{2}{*}{0.0153} & \multirow{2}{*}{24.5} & \multirow{2}{*}{0.0192} & \multirow{2}{*}{$\begin{array}{c}\text { Statistically } \\
\text { significant } \\
\text { difference }\end{array}$} \\
\hline & WOD & 4.6755 SD 1.8188 & 0.9573 & 0.7376 & & & & & & & \\
\hline \multirow{2}{*}{$\begin{array}{l}\text { Shoulder Pain } \\
\text { Rating }\end{array}$} & WD & 2.4727 SD 1.1637 & 0.9549 & 0.7064 & \multirow{2}{*}{0.9664} & \multirow{2}{*}{$\begin{array}{c}-0.2722- \\
2.2049\end{array}$} & \multirow{2}{*}{-1.6275} & \multirow{2}{*}{0.1193} & \multirow{2}{*}{37} & \multirow{2}{*}{0.1294} & \multirow{2}{*}{$\begin{array}{c}\text { No statistically } \\
\text { significant } \\
\text { difference }\end{array}$} \\
\hline & WOD & 3.4391 SD 1.5887 & 0.9450 & 0.5814 & & & & & & & \\
\hline
\end{tabular}

Table 2: Statistical test results on the primary outcome measures, tested in the group that successfully finished the study ( $n=11)$. WD='with device'; WOD='without device'. Detailed explanation of the statistical tests can be found in the "Data Analysis" section. 


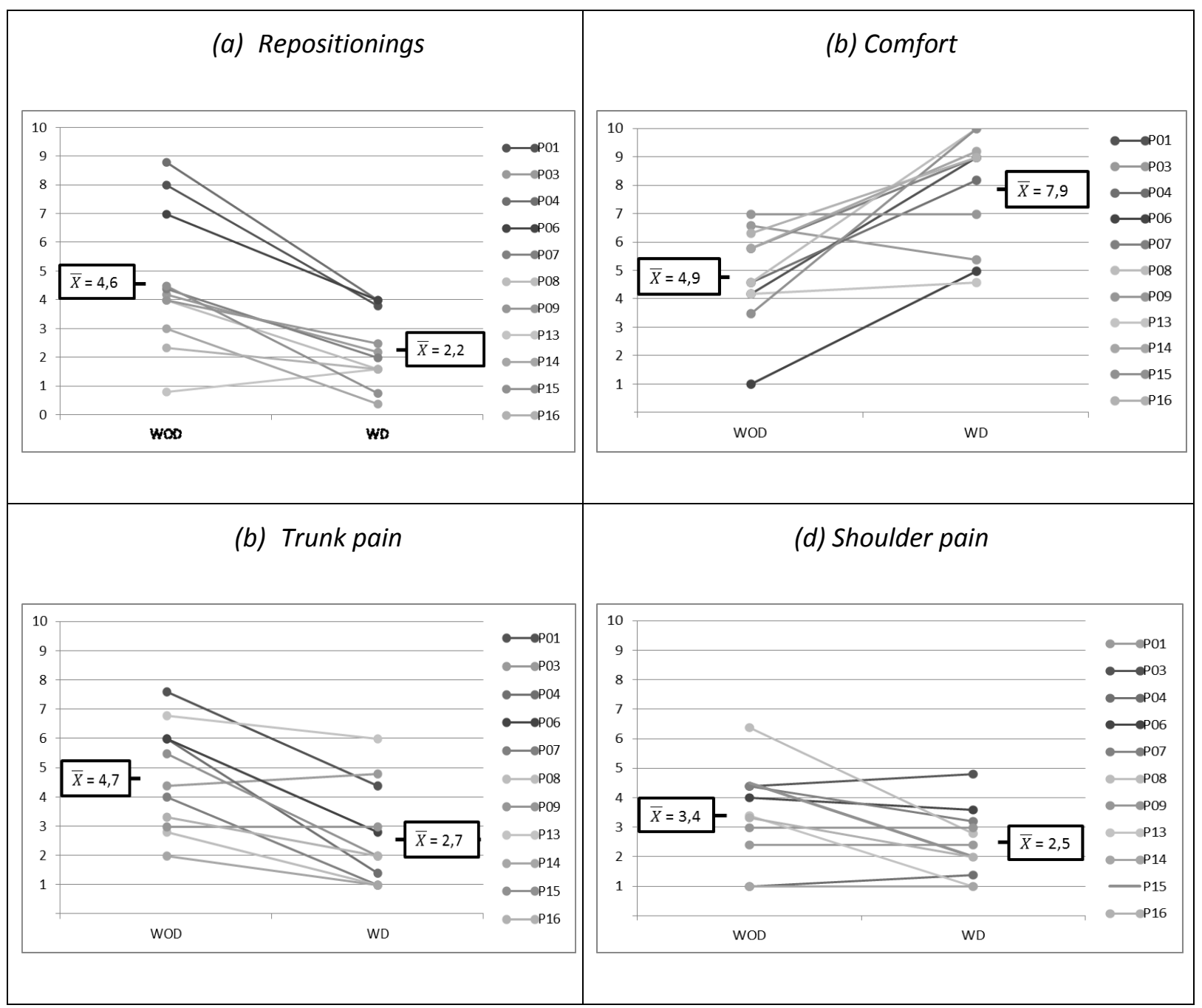

Figure 4: Individual group changes in the primary outcome measures, comparing regular care practice (WOD='without device') with the novel posture support (WD='with device'), within the same eleven successful users in Hospital Gorliz ( $n=11)$, alternatively undergoing both modalities of care, (a) number of re-positioning by caregivers during a day, (b) perceived comfort (c) perceived trunk pain, and (d) perceived shoulder pain. All four differences between WOD and WD condition were significant $(p<0.05)$ in paired testing of differences; for significance levels see Table 2.

As noted before, the WPSD appeared to be not suitable for one of the patients included in the study. This patient used the WPSD still for two days. Four other patients used the device conveniently, even though they did not complete the follow-up of five days because of reasons unrelated to the study or the device. Table 3 presents the results of these patients $(n=5)$. These are valid results, but could not be included in the overall statistics, because they did not fulfil the study design. Nevertheless, this group shows the same trends for all scores as the group that fully completed the study. 


\begin{tabular}{|l|c|c|}
\hline \multicolumn{2}{|c|}{ Parameter } & Experimental parameter value \\
\hline \multicolumn{2}{|c|}{} & Mean \pm Standard Deviation \\
\hline $\begin{array}{l}\text { Number of re- } \\
\text { positioning per half day }\end{array}$ & WD & 2.0667 SD 1.7504 \\
\cline { 2 - 3 } Comfort Rating & WOD & 3.6833 SD 0.3249 \\
\cline { 2 - 3 } & WD & 7.3000 SD 2.7749 \\
\hline \multirow{2}{*}{ Trunk Pain Rating } & WOD & 5.4000 SD 1.3874 \\
\hline \multirow{2}{*}{ Shoulder Pain Rating } & WD & 3.7000 SD 2.9496 \\
\cline { 2 - 3 } & WD & 4.3000 SD 2.1679 \\
\cline { 2 - 3 } & WOD & 1.9000 SD 1.0247 \\
\hline
\end{tabular}

Table 3: Results on the primary outcome measures, for the group that did not successfully finish the follow-up of five days for reasons unrelated to the study, but that could conveniently use the device $(n=5) . W D=$ 'with device'; WOD='without device'. Although statistical methods cannot be applied on this group, it is clear that the trends for all scores are exactly the same as in the group that completed the study.

\section{Secondary outcomes}

The overall ease of use was assessed by the professional caregivers that were operationally involved in using the device under study $(n=28)$, as 4.17 SD 0.78 out of 5 (with 1 very difficult to use, 5 very easy to use), scored on separate items as shown in table 4.

\begin{tabular}{|l|l|}
\hline Usability Item & $\begin{array}{l}\text { Mean } \pm \text { Standard Deviation } \\
(1=\text { very difficult / inconvenient } \quad . . .5=\text { very } \\
\text { easy / convenient })\end{array}$ \\
\hline Ease to put the device on the wheelchair & 4.31 SD 0.48 \\
\hline Ease to apply the device to the patient & 3.88 SD 0.81 \\
\hline Ease to apply vacuum to the device & 3.88 SD 0.81 \\
\hline Ease to perform a re-positioning & 2.94 SD 0.44 \\
\hline Ease to take out the patient from wheelchair & 5 SD 0 \\
\hline Hygiene & 5 SD 0 \\
\hline
\end{tabular}

Table 4: Scores on perceived usability of the WPSD under professional caregivers operationally involved in the study $(n=28)$.

The relatively low score on performing a re-positioning was explained as that a number of times it was needed to operate the valve, and re-apply a vacuum, make the device flexible and again apply a vacuum, which was considered as relatively cumbersome.

The functionality was assessed for all 16 patients that initially started the study, and not only for the 11 who completed the 5 days follow-up. The rigidity of the WPSD was considered adequate in the majority ( $81.25 \%)$ of cases. In the rest of cases $(18.75 \%)$ vacuum had to be applied repeatedly during operation to maintain functional rigidity. In none of the cases the rigidity was considered insufficient. The device could be adequately adapted to shape in $87.5 \%$ of cases. In two patients $(12.5 \%)$ the adaptation of shape was considered overall insufficient, one was a very slim patient, that also had to stop the study because of an unrelated back lesion, whereas in the other patient the device did not achieve a functional performance. The device did allow a transfer without difficulties of patients into and out of the wheelchair in all cases (100\%). The amount of time needed to apply and adjust the device was perceived as low in $56 \%$ of cases and as medium in $44 \%$ of cases, and as high in $0 \%$ of cases. 


\section{Discussions and conclusion}

In this article, we presented a novel Wheelchair Posture Support Device (WPSD) to assist sub-acute post stroke patients in maintaining a correct upright sitting posture in a wheelchair during their hospitalization. A user-centred design process and a novel material technology referred to as Varstiff $^{\mathcal{O}}$, were employed to achieve the demanded specifications.

A prototype of the WPSD was clinically validated with 16 patients in Hospital Gorliz, a rehabilitation centre for stroke patients of the Basque Public Health System. To carry out the clinical study, it was necessary to take into consideration some limitations. A first limitation was that the Wheelchair Posture Support Device was compared only with the conventional care practice in Hospital Gorliz since no other products similar to WPSD were available. To our knowledge, only cushions and supports specifically designed for one single user, or backward-tilting wheelchairs are existing solutions that could provide comparable results. However, these solutions are high-end and expensive solutions, and typically not used in the target group of the WPSD, that only stays for a limited time in a hospital setting. When feasible alternative solutions are available or identified, a study similar to the work presented here could identify the relative performance of WPSD versus that specific product. A second limitation of the study was that the WPSD was designed for the basic wheelchair in use in Hospital Gorliz (figures 2 and 3), and was only validated with this type of wheelchair, which means that it may not be possible to install the WPSD in other kinds of wheelchair without adaptations to the design.

Notwithstanding aforementioned limitations, the clinical validation clearly demonstrated that the developed Wheelchair Posture Support Device, based on Varstiff ${ }^{\odot}$ technology, is able to improve sitting posture of post-acute stroke patients when seated in a wheelchair during hospitalized daycare, and leads to a reduction of caregiver involvement in assuring proper sitting posture by $52 \%$, when applied in a standard real care environment, according to the reduction of number of times a patient has to be re-positioned during a set time duration. The WPSD device was rated as comfortable and as reducing pain complaints (only statistically significant for trunk pain, not for shoulder pain), rated by the patients (or caregivers when not feasible). As secondary outcome, the professional caregivers subjectively rated the device as highly easy to use, and positively rated the functionality of the device in the majority of patient cases. Only in one patient the WPSD was not applicable, since the device appeared to slide inside the wheelchair. However, relatively simple material improvements may prevent such sliding from happening, even in such cases where patients exercise a high force on the seating surface.

With these results, the WPSD provides an example that the Varstiff $^{\oplus}$ technology can be effectively applied for improving supports and assistances in stroke care, because of its typical capacity to be shaped in a flexible state according to the needs of a specific user, and to be switched to a rigid state once formed to a functional shape in order to provide proper support.

Additionally, the WPSD may be applicable to other user groups, outside of hospitalized post-acute stroke patients, for any patient group in which there is an apparent risk of poor sitting posture or falling out of the wheelchair, especially in groups that spend considerable time of the day seated.

In conclusion, the novel WPSD arises as a valuable system for the improvement in medical assistance of hospitalized wheelchair-bound post-stroke patients, by reducing pain and number of repositioning manoeuvres, which might be also applicable to any group of patients who need posture control. 


\section{Acknowledgements}

We would like to thank the following institutes, companies and persons for their support realizing this work:

- Hospital Gorliz; patients and care professionals involved in the study for their help, especially: physicians Susana Pinedo-Otaola and Pedro Tejada-Ezquerro.

- Textia Innovative Solutions SL; for coordinating the production of the prototype

- The FIK initiative; for funding the development of the Varstiff ${ }^{\odot}$ material technology

- Fundación Botín's 'Mind the Gap' program; for co-funding the design process of the WPSD

- Spherium Biomed; for co-funding the study with the WPSD

- Aitor Belloso of Tecnalia, for his support in the development of the WPSD

- Joe McIntyre of Tecnalia, for his support in improving the English writing style of the manuscript

- $\quad$ Maxime Bureau at Deflastrat SL; for the production of the prototype Varstiff ${ }^{\odot}$ trunk support

- Núria Coll at Núria Coll - Disseny Industrial; for the industrial design and production of the prototype vest and cushion

\section{Declaration of Interest}

The Varstiff ${ }^{\odot}$ material was invented and patented by Tecnalia, funded by the FIK initiative. For commercialization purposes, the rights of the Varstiff $^{\odot}$ material technology were transferred to Textia Innovative Solution.

The Wheelchair Posture Support Device, based on the Varstiff ${ }^{\complement}$ material, presented in this paper, may be commercialized through Textia Innovative Solutions.

The FIK initiative and Spherium Biomed have a specific equity in Textia Innovative Solutions.

BIOEF and Hospital Gorliz (Osakidetza), that mainly carried out and approved the validation study and its results, have no share or commercial interest in this technology or its commercialization.

\section{References}

1 Feigin V, Forouzanfar M, Krishnamurthi R, Mensah G, Connor M, Bennett D, Moran A, Sacco R, Anderson L, Truelsen T, et al. Global and regional burden of stroke during 1990--2010: findings from the Global Burden of Disease Study 2010. The Lancet. 2014;383(9913):245-255.

2 Keating M, Penney M, Russell P, Bailey E. Positioning and early mobilisation in stroke. Nursing times. 2011;108(47):16-18.

3 Hargroves D, Tallis R, Pomeroy V, Bhalla A. The influence of positioning upon cerebral oxygenation after acute stroke: a pilot study. Age and ageing. 2008;37(5):581-585.

4 Tyson S, Nightingale P. The effects of position on oxygen saturation in acute stroke: a systematic review. Clinical Rehabilitation. 2004;18(8):863-871.

5 Chatterton H, Pomeroy V, Gratton J. Positioning for stroke patients: a survey of physiotherapists' aims and practices. Disability \& Rehabilitation. 2001;23(10):413-421. 
6 Shumway-Cook A, Woollacott M. Motor control: translating research into clinical practice. Lippincott Williams \& Wilkins; 2007.

7 Chaves E, Cooper R, Collins D, Karmarkar A, Cooper R. Review of the use of physical restraints and lap belts with wheelchair users. Assistive technology. 2007;19(2):94-107.

8 Heart \& Stroke Foundation. A guide for stroke caregivers. [Internet]. 2013 [cited 2015 January 25]. Available from: "http://www.heartandstroke.on.ca/atf/cf/\%7B33C6FA68-B56B-4760-ABC6D85B2D02EE71\%7D/Final_\%20TipsAndTools_eng.pdf" .

9 Bureau M, Keller T, Veneman J, Vera C, inventors. Elemento con rigidez variable controlada por presion negativa. 2014. WO2014140389. Available from:

"https://www.google.es/patents/WO2014140389A1?cl=es" .

10 Bureau M, Jung JH, Keller T, inventors. Fitting element with controlled stiffness. 2011. WO2011079865. Available from: "https://www.google.es/patents/WO2011079865A1?cl=en".

11 Bureau M, Keller T, Perry J, Velik R, Veneman J. Variable stiffness structure for limb attachment. In: Rehabilitation Robotics (ICORR), 2011 IEEE International Conference on; 2011. p. 1-4.

12 Tilley AR, Anning J, Welles R. The Measure of Man and Woman: Human Factors in Design. Revised Edition. Wiley \& Sons; 2002.

13 Kalichman L, Ratmansky M. Underlying pathology and associated factors of hemiplegic shoulder pain. American Journal of Physical Medicine \& Rehabilitation. 2011;90(9):768-780.

14 Lindgren I, Jonsson A, Norrving B, Lindgren A. Shoulder pain after stroke A prospective population-based study. Stroke. 2007;38(2):343-348.

15 Hockenberry M, Wilson D, Wong D. Wong's Essentials of Pediatric Nursing9: Wong's Essentials of Pediatric Nursing. Elsevier Health Sciences; 2012.

16 Veneman J, Manterola I, Bureau M, Jung J. Varstiff, an innovative variable stiffness material, applied in a Wheelchair Positioning Device. In: TAR 2015: Technically Assisted Rehabilitation Conference, Berlin, Germany; 2015. 